\title{
Is a Basic Erosive Wear Examination (BEWE) reliable for recording erosive tooth wear on $3 \mathrm{D}$ models?
}

\section{Alaraudanjoki, Viivi}

2017-04

Alaraudanjoki , V , Saarela, H , Pesonen, R, Laitala, M-L , Kiviahde , H , Tjaderhane , L , Lussi , A , Pesonen, P \& Anttonen, V 2017 , ' Is a Basic Erosive Wear Examination (BEWE) reliable for recording erosive tooth wear on 3D models? ' , Journal of Dentistry, vol. 59 , pp. 26-32 . https://doi.org/10.1016/j.jdent.2017.02.001

http://hdl.handle.net/10138/236745

https://doi.org/10.1016/j.jdent.2017.02.001

publishedVersion

Downloaded from Helda, University of Helsinki institutional repository.

This is an electronic reprint of the original article.

This reprint may differ from the original in pagination and typographic detail.

Please cite the original version. 
Full length article

\title{
Is a Basic Erosive Wear Examination (BEWE) reliable for recording erosive tooth wear on 3D models?
}

\author{
Viivi Alaraudanjoki ${ }^{a}{ }^{\text {,* }}$, Henna Saarela ${ }^{a}$, Reetta Pesonen ${ }^{a}$, Marja-Liisa Laitala ${ }^{a}$, \\ Heikki Kiviahde ${ }^{\mathrm{a}}$, Leo Tjäderhane ${ }^{\mathrm{a}, \mathrm{b}, \mathrm{c}}$, Adrian Lussi ${ }^{\mathrm{d}}$, Paula Pesonen ${ }^{\mathrm{b}}$, Vuokko Anttonen ${ }^{\mathrm{a}, \mathrm{b}}$ \\ ${ }^{a}$ Research Unit of Oral Health Sciences, University of Oulu, PO Box 5281, 90014, Finland \\ ${ }^{\mathrm{b}}$ Medical Research Center Oulu, Oulu University Hospital and University of Oulu, Kajaanintie 54, 90029 Oulu, Finland \\ ' Department of Oral and Maxillofacial Diseases, University of Helsinki and Helsinki University Hospital, Helsinki, Finland, Kasarmikatu 11-13, 00029 Helsinki, \\ Finland \\ ${ }^{\mathrm{d}}$ Department of Preventive, Restorative and Pediatric Dentistry, University of Bern, Freiburgstrasse 7, CH-3010 Bern, Switzerland
}

\section{A R T I C L E I N F O}

\section{Article history:}

Received 31 October 2016

Received in revised form 31 January 2017

Accepted 3 February 2017

\section{Keywords:}

Erosive wear

BEWE

Validation

3D model

Assessment

Detection

\begin{abstract}
A B S T R A C T
Objectives: To assess the reliability of the BEWE index on 3D models and to compare 3D-assessed erosive tooth wear scores with clinically detected scores.

Methods: In total, 1964 members of the Northern Finland Birth Cohort 1966 participated in a standardized clinical dental examination including the Basic Erosive Wear Examination (BEWE) and dental 3D modelling at the age of $45-46$ years. Of those examined, 586 were randomly selected for this study. 3D models were assessed using the same BEWE criteria as in the clinical examination. Calculated kappa values as well as the prevalence and severity of erosive wear according to the clinical examination and 3D models were compared. Re-examinations were performed to calculate intra- and inter-method and -examiner agreements.

Results: The BEWE index on 3D models was reproducible; the mean intra- and inter-examiner agreement were 0.89 and 0.87 , respectively, for sextant level, and 0.64 and 1 , respectively, for BEWE sum scores. Erosive tooth wear was recorded as more severe in $3 \mathrm{D}$ models than in the clinical examination, and intermethod agreement was 0.41 for severe erosive wear (BEWE sum>8). The biggest inter-method differences were found in upper posterior sextants.

Conclusions: The BEWE index is reliable for recording erosive tooth wear on 3D models. 3D models seem to be especially sensitive in detecting initial erosive wear. Additionally, it seems that erosive wear may be underscored in the upper posterior sextants when assessed clinically. Due to the nature of 3D models, the assessment of erosive wear clinically and on 3D models may not be entirely comparable.

Clinical significance: 3D models can serve as an additional tool to detect and document erosive wear, especially during the early stages of the condition and in assessing the progression of wear. When scoring erosive wear clinically, care must be taken especially when assessing upper posterior sextants.
\end{abstract}

(c) 2017 Elsevier Ltd. All rights reserved.

\section{Introduction}

Erosive tooth wear results from the combined effect of chemical erosion caused by acids and the mechanical wear of acid-softened enamel by attrition and abrasion [1]. Owing to this multifactorial

\footnotetext{
* Corresponding author.

E-mail addresses: viivi.alaraudanjoki@oulu.fi (V. Alaraudanjoki),

henna.saarela@student.oulu.fi (H. Saarela),reetta.pesonen@student.oulu.fi (R. Pesonen), marja-liisa.laitala@oulu.fi (M.-L. Laitala), heikki.kiviahde@oulu.fi (H. Kiviahde), leo.tjaderhane@oulu.fi (L. Tjäderhane), adrian.lussi@zmk.unibe.ch (A. Lussi), paula.pesonen@oulu.fi (P. Pesonen), vuokko.anttonen@oulu.fi (V. Anttonen).
}

etiology, erosive wear can manifest itself in different ways and early diagnosis is particularly challenging [2]. Currently, there are a large number of different grading scales used for diagnosis; among them quite recently introduced Basic Erosive Wear Examination (BEWE) [3]. In the four-step BEWE classification, each surface of a tooth is examined and registered separately but recorded according to sextant; the most damaged tooth surface represents the BEWE score of a sextant (from 0 to 3 ). In class 0 , no signs of erosive wear are observed, whereas class 1 represents initial loss of surface texture. In class 2 , erosive lesions are more pronounced and there is hard tissue loss less than $50 \%$ of the surface area. In class 3 , hard tissue loss covers more than $50 \%$ of the surface area. The patient BEWE score is the sum score of the sextants (from 0 to 18). 
The BEWE has been developed by internationally recognized experts in the field and is intended to be an internationally accepted, standardized and validated index and a simple tool for clinical practice [3].

Studies concerning the reliability and repeatability of the BEWE index are rare, but moderate reliabilities have been reported $[4,5]$ and the BEWE has been approved for clinical and epidemiological use. The BEWE was originally developed to be suitable also for grading dental casts [3], but to date there are no studies assessing the reliability of BEWE on either dental casts or digital 3D models. However, BEWE has been shown to be suitable for evaluating erosive wear on photographs [6].

Irrespective of the grading scale or method used, erosive tooth wear appears to be challenging to detect in its initial stages [2,7]. Concerning tooth wear assessment in general, conventional clinical examination has even been suggested to be the least sensitive when compared to more sophisticated methods [8], including recording from high-resolution 3D modelling. However, it has been shown that initial wear is hard to distinguish also on study casts [9]. 3D models have been used clinically in orthodontics, oral surgery and implant treatments for years. More recently, computer-aided design and manufacturing, referred to as CADCAM-technology, has become increasingly popular in designing and manufacturing restorations, crowns and fixed prosthodontics. In general, CAD-CAM-technology and 3D models are expected to improve implementation of medical treatments and documentation of treatment outcomes [10]. This technology might also be useful in tooth wear assessment and treatment planning and monitoring. It has even been proposed that comparing sequential $3 \mathrm{D}$ models is the most accurate method for measuring tooth wear progression [11]. However, no epidemiologic studies concerning the use of 3D models in erosive tooth wear assessment have been reported.

The aim of this study was to assess whether classifying erosive tooth wear on 3D models using the BEWE index is reliable and whether erosive wear scores on 3D models are comparable with clinical findings. The hypothesis was that the BEWE index is reliable in the assessment of erosive tooth wear on 3D models, and that 3D models are suitable for detecting erosive tooth wear.

\section{Materials and methods}

\subsection{Study population}

The study population was a part of the Northern Finland Birth Cohort 1966 (NFBC 1966), initially comprising all 12,058 children whose expected time of delivery was in the year 1966 [12]. The cohort has been evaluated regularly since birth by means of health questionnaires and clinical examinations [12]. In connection with the 46-year follow-up survey (2012-2014), a subgroup of NFBC 1966 (a total of 3181 persons currently living in the city of Oulu or within $100 \mathrm{~km}$ of Oulu, including rural areas) was invited by mail to participate in clinical oral examinations. Examination protocol is more precisely described on our previous paper [4]. Participation in the study was voluntary and 1962 subjects agreed to participate. One-third of this original subgroup was randomly selected to comprise the present study population $(\mathrm{N}=600)$.

\subsection{Clinical examination}

Clinical dental examinations were performed between April 2012 and June 2013. Examinations were carried out supine in a dental chair with modern equipment and optimal lightning with a probe, oral mirror and WHO ball pointed gingival probe. The teeth were blow-dried using a three-in-one syringe before assessment. In the clinical examination, the BEWE index (0-3) was used to register erosive tooth wear in every sextant according to the original BEWE definitions [3]. All tooth surfaces were examined and the highest score for each sextant was recorded. The BEWE scoring criteria are presented in Table 1 . The examiners were advised not to include surfaces with only wedge-shaped defects or flat attritional facets in the assessment, if no signs of erosion were present. Erosive tooth wear could not be recorded if there were extensive restorations or prosthetic work covering the entire tooth surface. Wisdom teeth were excluded from the assessment.

\subsection{Validation in clinical examination of erosive wear}

A dentist specialized in the field of cariology $(\mathrm{VuA})$ instructed and calibrated seven dentists with respect to the clinical examination, including the erosive wear examination. The instruction comprised lectures on the use of the BEWE index to ensure that all examiners would have similar theoretical knowledge. Criteria for classifications were presented as a PowerPoint presentation on a screen using a PC and data projector. Calibration was repeated every third month. The criteria for the BEWE classifications were available to the examiners throughout the study in written and graphical form in the examination room. A senior dentist and researcher (MLL), familiar with the study protocol and criteria, acted as a gold standard and ensured that the examiners followed the study protocol precisely throughout the entire field study. To assess the intra-examiner agreement, the examiners re-examined one quadrant of an average of 5 patients approximately one month after the first examination. To assess the inter-examiner agreement, the gold standard examiner (MLL) reexamined one quadrant of an average of 10 patients from each examiner.

\subsection{D models}

On the clinical examination day, 3D models were obtained by dental hygienist trained by the manufacturer using an iTero 3D scanner (Cadent, San Jose, CA, USA). Models were scanned and stored unrevised in the server of the cohort. The 3D models were assessed on a PC screen (screen information) using 3Shape Ortho Analyzer ${ }^{\mathrm{TM}}$ software. Erosive tooth wear was assessed using the BEWE index and the same scoring criteria as in the clinical examination (Table 1). In the software, 3D models were yellow with different degrees of shading. It was possible to zoom and rotate the models while analyzing; thus it was possible to inspect models from different angles. For analyzing the 3D models, the gold standard examiner (MLL) and the instructor with regard to the clinical examination (VuA) trained three examiners (two fourthyear dental students with more than one year clinical experience (RP, HS) and one dentist (ViA)), using the same basis of instruction as in the clinical examination. The examiners were calibrated first through the coordinated analysis of 3D models until agreement was reached regarding the severity of erosive changes between themselves and with the gold standard examiner of the clinical examination. Analyses were performed over one week at the same location with the same lightning. Analyses were first timed to maximize the sample size in a limited period of time. Two fourth-

Table 1

Definitions of the Basic Erosive Wear Examination (BEWE) scores.

\begin{tabular}{ll}
\hline BEWE score definition \\
\hline 0 & No erosion \\
1 & Initial loss of surface texture \\
$2^{\mathrm{a}}$ & Distinct defect, hard tissue loss less than $50 \%$ of the surface area \\
$3^{\mathrm{a}}$ & Hard tissue loss more than $50 \%$ of the surface area \\
\hline
\end{tabular}

a Dentine is often involved. 
year dental students analyzed all 600 3D models together, and the dentist (ViA) acted as the gold standard examiner for reexaminations and was available in the examination room throughout the study. The criteria for the BEWE classifications were available to the examiners in written and graphical form in the analyzing room. To assess intra-examiner agreement 19 (144 sextants) randomly picked 3D models were re-examined during the analysis of all 600 models. To assess inter-examiner agreement 11 (66 sextants) randomly chosen 3D models were re-examined after analyzing all 600 3D models. The subjects were randomized by choosing every twentieth person from a list in which the subjects were sorted according to their project ID. The reexaminations were performed at the same time at the same space, and the students were allowed to discuss using a few words with each other while deciding the score.

\subsection{Statistics}

To describe and analyze the prevalence and severity of erosive tooth wear according to the clinical examination and analyzed 3D

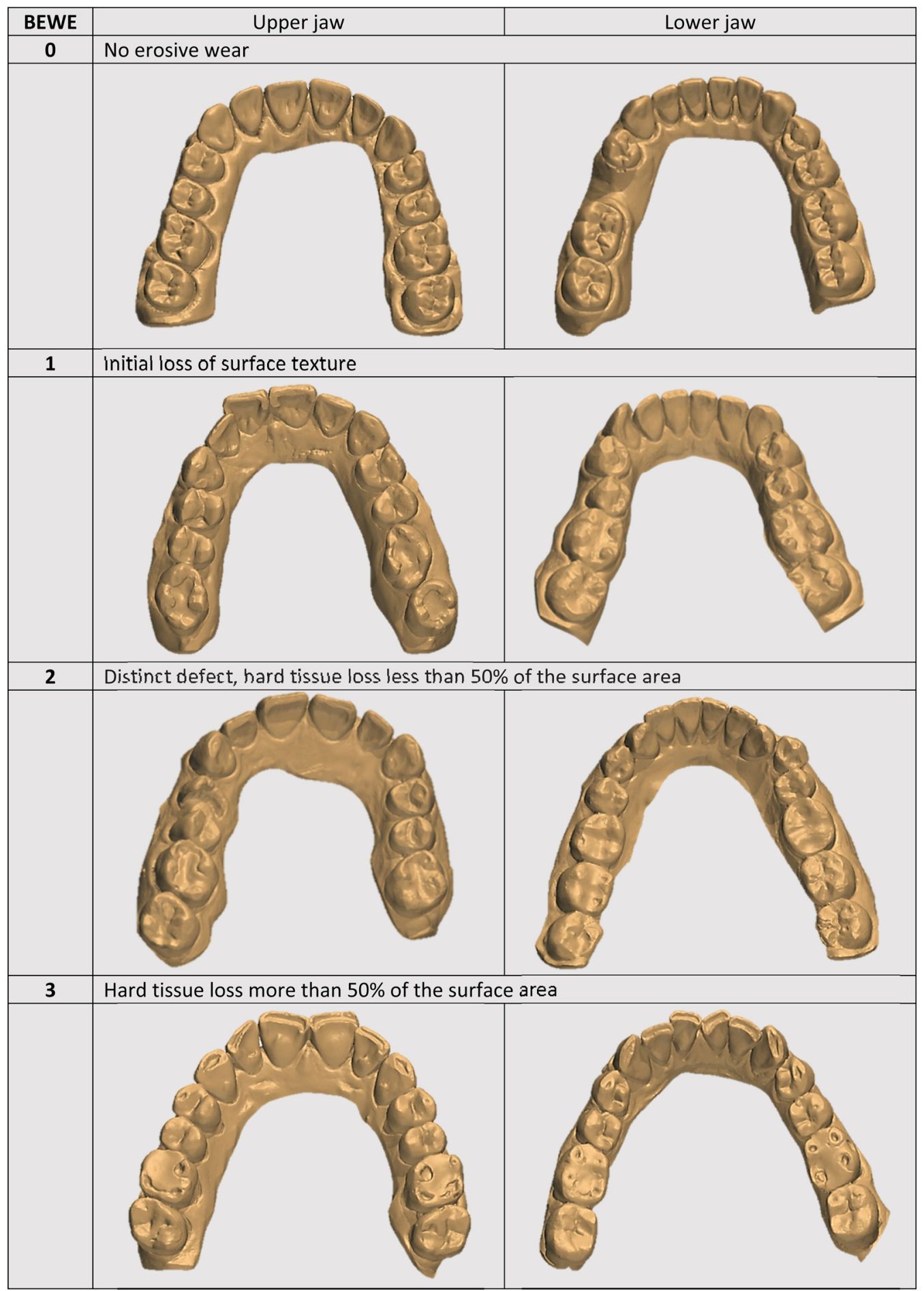

Fig. 1. Different stages of erosive tooth wear according to BEWE index in 3D models. 
models, the sum scores of the BEWE index were calculated and interpreted as follows (adjusted from the original scoring system [3] by combining the two highest risk groups): BEWE sum score 0 $2=$ no or only mild erosive tooth wear with no treatment needed; BEWE sum score 3-8= moderate erosive tooth wear, treatment needed; and BEWE sum score 9-18=severe erosive tooth wear, treatment needed.

Descriptive statistics such as frequencies, distributions, means and standard deviations were calculated. The kappa coefficient and percentage agreement was calculated to analyze inter- and intraexaminer as well as inter-method agreements. Kappa values were interpreted as suggested by Landis and Koch [13]. The intermethod difference between mean BEWE outcomes per sextants was analyzed using the Wilcoxon signed rank test and the mean BEWE sum score using a paired sample $t$-test. The differences of the BEWE sum score distributions between the methods were tested using the chi-square test and Fisher's exact test. A $P$ value $<0.05$ was considered statistically significant. All statistical analyses were performed using SPSS (version 22.0, SPSS, Inc., Chicago, IL, USA).

\section{Results}

In total, the study analyzed 600 3D models, of which 599 were included in the analyses ( 1 was missing owing to unidentified ID). Of these, 586 had corresponding clinical data of erosive wear. Fig. 1 illustrates the different stages of erosive tooth wear in 3D models. Altogether, erosive wear was recorded as more severe in the $3 \mathrm{D}$ models than in the clinical examination.

\subsection{BEWE scores based on sextants}

The distribution of BEWE scores per sextant is shown in Table 2. According to both methods, anterior sextants were the most affected by erosive wear, with mean BEWE scores close to 1 both in the 3D models and in the clinical examination. The biggest intermethod differences in mean scores existed in upper posterior sextants, and the smallest in lower posterior sextants. The intermethod difference between the mean BEWE scores was statistically significant $(\mathrm{p}<0.001)$ in all sextants, except for lower left posterior sextant $(\mathrm{p}=0.231)$.
Table 3

Distribution of BEWE sum scores $(0-2,3-8,9-18)$ according to 3D models and clinical examination.

\begin{tabular}{cccc}
\hline BEWE sum score & $\begin{array}{c}\text { 3D models } \\
\mathrm{n}(\%)\end{array}$ & $\begin{array}{c}\text { Clinical examination } \\
\mathrm{n}(\%)\end{array}$ & p-value \\
\hline $0-2$ & $186(31.1)$ & $318(53.1)$ & $<0.001$ \\
$3-8$ & $353(58.9)$ & $230(38.4)$ & $<0.001$ \\
$9-18$ & $60(10.0)$ & $38(6.3)$ & 0.02 \\
Total & $599(100)$ & $585(100)$ & \\
\hline
\end{tabular}

* p-value for the differences of the BEWE sum scores between the methods.

\subsection{BEWE sum scores}

The mean (SD) BEWE sum score calculated from the 3D models and the clinical examinations were 4.3 (3.17) and 3.3 (3.23), respectively $(\mathrm{p}<0.01)$. The inter-method difference between the BEWE sum categories was statistically significant $(\mathrm{p}<0.001)$ in all sum score categories (Table 3 ) - the percentage difference was the smallest for severe erosive wear (4\%) and biggest for no to mild erosive wear (22\%) (Table 3 ). Only $6 \%$ of the examined subjects were free of erosive wear according to the 3D models, whereas $26 \%$ were free from erosive wear according to the clinical examination.

\subsection{Reproducibility in $3 D$ models}

The mean intra-examiner agreement between sextants was excellent (Table 4). The lowest kappa value was found between upper anterior and lower right posterior sextants and the highest between left posterior sextants. For BEWE sum scores, the intraexaminer agreement was fair-to-good, percentage agreement reaching 95\%. The mean inter-examiner agreement was also excellent. The lowest value was found for upper left posterior sextant and the highest for upper anterior and lower posterior sextants. For BEWE sum scores the inter-examiner agreement was 1 (cut-off point BEWE sum $>8$ ). Considering erosive wear of any degree, intra- and inter-examiner agreements were lower, but still fair-to-good (Table 4).

\subsection{Reproducibility of the clinical examination on sextant level}

The mean intra- and inter-examiner agreements between sextants were excellent (Table 4). The lowest kappa value was found for lower left posterior sextant. In all other sextants, the level of agreement was approximately the same. Considering erosive

Table 2

Means and distributions of BEWE scores per sextant according to 3D models and clinical examinations.

\begin{tabular}{|c|c|c|c|c|c|c|c|}
\hline \multirow[t]{2}{*}{ BEWE score } & \multicolumn{7}{|l|}{ Sextant } \\
\hline & $\begin{array}{l}\text { Upper posterior } \\
\text { right } \mathrm{n}(\%)\end{array}$ & $\begin{array}{l}\text { Upper anterior } \\
\mathrm{n}(\%)\end{array}$ & $\begin{array}{l}\text { Upper posterior } \\
\text { left } \mathrm{n}(\%)\end{array}$ & $\begin{array}{l}\text { Lower posterior } \\
\text { left } \mathrm{n}(\%)\end{array}$ & $\begin{array}{l}\text { Lower anterior } \\
\mathrm{n}(\%)\end{array}$ & $\begin{array}{l}\text { Lower posterior } \\
\text { right } \mathrm{n}(\%)\end{array}$ & $\begin{array}{l}\text { Total } \\
\mathrm{n}(\%)\end{array}$ \\
\hline \multicolumn{8}{|l|}{ 3D models } \\
\hline Mean & 0.69 & 1.00 & 0.69 & 0.46 & 0.94 & 0.53 & 0.72 \\
\hline 0 & $283(47.2)$ & $196(32.7)$ & $274(45.7)$ & $398(66.4)$ & $212(35.4)$ & $362(60.4)$ & $1725(48.0)$ \\
\hline 1 & $230(38.4)$ & $248(41.4)$ & $252(42.1)$ & $136(22.7)$ & $233(38.9)$ & $166(27.7)$ & $1265(35.2)$ \\
\hline 2 & $74(12.4)$ & $113(18.9)$ & $58(9.7)$ & $53(8.8)$ & $129(21.5)$ & $59(9.8)$ & $486(13.5)$ \\
\hline 3 & $12(2.0)$ & $42(7.0)$ & $15(2.5)$ & $12(2.0)$ & $25(4.2)$ & $12(2.0)$ & $118(3.3)$ \\
\hline \multicolumn{8}{|c|}{ Clinical examination } \\
\hline Mean & 0.43 & 0.82 & 0.41 & 0.44 & 0.76 & 0.42 & 0.55 \\
\hline 0 & $372(62.1)$ & 185 (30.9) & $378(63.1)$ & $366(61.1)$ & $225(37.6)$ & $375(62.6)$ & $1901(54.1)$ \\
\hline 1 & $178(29.7)$ & $322(53.8)$ & $177(29.5)$ & $186(31.1)$ & $277(46.2)$ & $174(29.0)$ & $1374(39.1)$ \\
\hline 2 & $34(5.7)$ & $76(12.7)$ & $29(4.8)$ & $27(4.5)$ & $82(13.7)$ & $34(5.7)$ & $282(8.0)$ \\
\hline 3 & $1(0.2)$ & $3(0.5)$ & $2(0.3)$ & $5(0.8)$ & $2(0.3)$ & $2(0.3)$ & $15(0.4)$ \\
\hline
\end{tabular}

The inter-method difference between mean scores was statistically significant in all sextants, except for sextant 4 . 
wear of any degree, the agreements were again lower; from poor to fair-to-good. Percentage agreement was above $70 \%$.

\subsection{Inter-method agreement}

On a sextant level, the mean inter-method agreement was poor (Table 4), percentage agreement ranging between $75 \%$ and $90 \%$. The lowest level of agreement was for lower anterior sextant. With respect to BEWE sum scores, the inter-method agreement was fairto-good, percentage agreement being $91 \%$. Of those subjects analyzed as having a BEWE sum score $0-2$ according to the 3Dmodels $(n=182), 73 \%$ had the same level of detection clinically. On the other hand, of those subjects analyzed as having a BEWE sum score $0-2(n=318)$ clinically, $42 \%$ had the same level of detection according to the 3D models.

Considering the clinical examination as a gold standard, the sensitivity and specificity of the outcome using 3D models with treatment need as the cut-off point were $82 \%$ and $42 \%$, respectively. If severe erosive wear was used as the cut-off point, the sensitivity was $58 \%$ and specificity was $93 \%$

\section{Discussion}

The use of the BEWE index on 3D models was reproducible, fast and easy to adapt. The BEWE index appears to be reliable for erosive wear assessment using 3D models. 3D models were convenient to analyze because they could be inspected from different perspectives and be zoomed. Direct intraoral 3D scanning was not considered difficult. The method was sensitive, especially for finding those in need for treatment. Due to the high reproducibility of the method, 3D models might also be useful for assessing the progression of erosive tooth wear. Even so, a low level of agreement between 3D models and clinical examinations and relatively low sensitivity for severe erosive wear should be addressed. According to present results, it seems that assessing erosive wear using 3D models and clinical examination are not fully comparable, and 3D modelling is not to replace the clinical assessment of erosive wear.

In recent years, relevant improvements have been achieved in digital dentistry. The CAD-CAM technique and 3D models are expected to have a large potential on tooth wear studies [14]. No epidemiological studies of the assessment of erosive wear on 3D models have been published so far. However, there is evidence that 3D measuring techniques are more accurate in quantifying and monitoring tooth wear than clinical examinations [8], and 3D measuring techniques may even be the most accurate methods for evaluating restoration wear indirectly [15]. At the beginning of the present study, the hypothesis was that erosive wear assessment using 3D models is challenging, especially because of the unicolor design of the model. However, it became evident that since even the smallest morphologic changes of surfaces were relatively easy to detect. Indeed, a 3D scanner is capable of detecting volume loss from etching wear even after $1 \mathrm{~min}$ exposure to acid [16]. The magnification and accuracy of 3D models as well as the ability to view models from different perspectives, without any time limit, is likely to facilitate the detection of erosive wear. In addition, the absence of saliva and other patient-related-factors probably simplifies the detection of erosive wear on 3D models. On the other hand, it must be remembered that, despite that 3D models seem to be extremely precise, there may be several flaws with the stitching and smoothing data gathering processes, which are not yet fully explored. However, it seems that the increased sensitivity in early wear detection using 3D models may be one reason for the lower levels of agreement with the clinical examination.

For assessing dental erosion, low to moderate agreements between indirect methods, such as photographs and dental casts, and clinical examinations have been reported previously [6,9]. In our opinion, the low inter-method reproducibility in this study is basically not erosion index-dependent because the BEWE class 1 includes all of the early erosive changes. In fact, this study confirms that erosive tooth wear is challenging to detect clinically, especially in its very early stages. However, due to the nature of the $3 \mathrm{D}$ models, it must be questioned if the same BEWE sum score cut-offvalues are valid for BEWE scores from 3D models and clinical examination, since BEWE is initially designed for clinical use. The fact that the $3 \mathrm{D}$ models seem to be very precise, may also explain the low specificity of the 3D models with respect to finding those not in need for treatment. Nevertheless, the sensitivity of the 3D models in finding those with severe erosive wear was relatively low. At times it was difficult to distinguish even between a worn filling and an eroded tooth surface. In addition, the optical properties of enamel are impossible to assess on 3D models. For severe cases, the high specificity but relatively low sensitivity of 3D models highlights the need for clinical examination, and 3D modelling might be more useful in assessing the progression of wear.

While the classification of the BEWE index does not include distinguishing between enamel and dentinal lesions [3], the index is well suited for this intention and enables the use of 3D models in erosion assessment - a feature that many of the erosion indices lack. The BEWE index itself has been validated and shown to be acceptable for recording tooth wear and for scoring its severity clinically $[5,7,17,18]$ as well as on photographs [7]. In our study, the clinical examiners had difficulty in differentiating between intact enamel and initial loss of surface texture. However, with respect to more advanced wear, the clinical examination performed well. Clinical assessment of occlusal and non-occlusal tooth wear has previously been reported to be more reliable than grading dental casts [9]. However, according to the present study, the advantage of 3D models seems to be that they may be more sensitive especially in finding initial erosive wear on natural tooth surfaces when compared to clinical examination.

In both the clinical examination and 3D models, anterior sextants were the most affected by erosive wear; the upper

Table 4

Reproducibility of the two methods and inter-method agreement based on the mean kappa values using different cut-off points.

\begin{tabular}{|c|c|c|c|}
\hline \multirow[t]{2}{*}{ Method } & \multicolumn{2}{|c|}{ Mean kappa values (sextant) } & \multirow{2}{*}{$\frac{\text { Mean kappa value (sum) }}{\text { cut-off BEWE sum }<8}$} \\
\hline & cut-off BEWE 2 (min, max) & No cut-off (min, max) & \\
\hline \multicolumn{4}{|l|}{ 3D models } \\
\hline Intra-examiner & $0.89(0.78-1)$ & $0.71(0.36-0.92)$ & 0.64 \\
\hline Inter-examiner & $0.87(0.62-1)$ & $0.72(0.45-0.86)$ & 1 \\
\hline \multicolumn{4}{|c|}{ Clinical examination } \\
\hline Intra-examiner & $0.98(0.84-1)$ & $0.46(0.13-1)$ & - \\
\hline Inter-examiner & 0.81 & 0.30 & - \\
\hline Inter-method & $0.31(0.23-0.39)$ & - & 0.41 \\
\hline
\end{tabular}


anterior sextant was slightly more affected than the lower one. The predominance of anterior sextants has also been reported previously [19]. Erosive wear in anterior sextants was again classified as more severe in 3D models than in clinical examinations. The lowest inter-method agreement was found for lower anterior sextant. For this, one explanation may be that in the clinical examination protocol it was emphasized to not include any attritional facets of anterior sextants in the BEWE. Since different wear mechanisms rarely act alone and may be difficult to distinguish, especially concerning anterior regions, some registration bias seems possible. Possibly the inclusion/exclusion criteria definitions concerning anterior regions should have been even more precise to avoid this problem. The causes of the intra-method differences in detecting erosive wear from lower posterior regions can, however, only be speculated on. The enamel is thinner in lower molars compared to upper molars and thinnest at the mesiobuccal cusp of the lower first molar [20,21], and hence the process appears to be more advanced there. Interestingly, the fourth and sixth sextant were similarly classified despite the method used. This might indicate that the detection of erosive wear is, overall, clinically easiest in the posterior parts of the lower jaw. These aspects may explain the finding that lower first molars are the teeth where erosive wear occurs most often [22]. Intermethod differences concerning upper posterior and anterior sextants were the main reasons for the differences in the BEWE sum scores between 3D models and clinical examination.

Upper posterior sextants exhibited significantly more erosive wear in 3D models when compared to lower posterior sextants (mean BEWE index 0.69 (upper) vs. 0.50 (lower)). In clinical examinations no such difference was found (mean BEWE index 0.42 (upper) vs. 0.43 (lower)). We speculate that this inter-method difference concerning posterior sextants may be due to the poorer visibility and light when clinically assessing upper posterior teeth compared to lower ones. Additionally, in this age group the first lower molars are often restored because of caries, and signs of erosive wear are not easily detectable. This may also partly explain the lower intra-method reproducibility of the lower posterior sextants. The predominance of the upper posterior sextant has not been reported previously. Based on these observations, detecting erosive wear clinically in upper posterior sextants must be emphasized.

This study is the first to assess the reliability of the BEWE index on 3D models as well as to compare erosive wear assessment using outcomes of analyses of 3D models and clinical examination. Using the same examiners to assess both methods throughout the study would have been the optimum design, however, this was not possible. To avoid shortcomings due to this, the examiners of the 3D models were calibrated by the same expert that had trained and calibrated the clinical examiners. In addition, the gold standard of the clinical examination was also involved in the calibration process to make sure the examiners of the 3D models would have similar inclusion and exclusion criteria as well as knowledge of BEWE index. During the 3D calibration process, it became apparent that understanding the morphologic features of healthy as well as restored teeth is essential in detecting erosive wear on 3D models. Initially, dental students had problems in picturing what normal variation can be and what erosive wear looks like, as well as, for example, in distinguishing the borderlines of restorations. Thus choosing relatively unexperienced examiners, might have produced bias in the study. Good repeatability between the gold standard and the undergraduate examiners, however, does not support this assumption.

Overall, the lack of a gold standard is the main difficulty in designing a study of this kind. It could also be argued that the BEWE index could be more precise in the description of classes, since the word "initial" and "distinct" can be understood in different ways. Even illustrations would be desirable in order to guide dentists through the education and calibration processes. In that way, the BEWE index would be more likely to be interpreted in the same way worldwide. Another limitation specifically concerning the inter-method approach is the difference between the clinical examination and the 3D model analysis protocol. The clinical examination was one part of a large-scale cohort study with several other variables to be registered, and it was also performed by seven dentists. On the other hand, the 3D models were analyzed only for erosive wear by two fourth-year dental students together over one week without any pressure concerning time. Such issues may cause bias in the outcome of inter-method agreement. In addition, owing to the re-examination protocol in the clinical examination, no reproducibility with respect to BEWE sum scores could be analyzed in the clinical examination. Added to that, 3D models seemed to be surprisingly precise, thus producing a challenge in the initial aim of validating the BEWE index. Concerning that much more erosive lesions are to be found when using 3D modelling, it is hard to say if there is a risk for overtreatment or a chance to prevent the lesions at the very right time. In our opinion, at least for younger age groups, finding erosive lesions as soon as possible gives dental professional more time to prevent the condition. Additionally, 3D models showing even the smallest erosive lesions, can also be used in motivating the patients to change the harmful erosive habits into a more healthy ones.

Analyzing 3D models over limited period of time is a major advantage in assessing the reproducibility of BEWE using 3D models. The large sample size overall strengthens the statistical power of this study. Additionally, this study supports the suggestion by Haketa et al. [23] that it is not very difficult to calibrate two relatively inexperienced examiners. Based on our observation, we expect that it will be quite simple to train general practitioners in using the BEWE index to enhance the early detection and management of erosive wear. This study shows that 3D models could serve as an additional assessment tool to detect and document erosive tooth wear especially in the early stages of the condition. Previously considered as too expensive, uncomfortable and time-consuming to use, these sophisticated digital techniques are now becoming cheaper, easier and faster to operate. Future studies are needed on the use of 3D models and especially in assessing the progression of erosive wear, since it seems to show promise for that as well.

\section{Conclusions}

The BEWE index appears to be reproducible in the assessment of erosive wear using 3D models. The results of the present study indicate that the supplementary use of 3D models could offer a substantial advantage compared with a clinical examination alone when assessing and monitoring erosive tooth wear. It also seems that erosive wear scoring may be underestimated clinically on the upper posterior sextants.

\section{Conflicts of interest}

None.

\section{Acknowledgements}

We thank the late Professor Paula Rantakallio (launch of NFBC 1966), the participants in the 46y study and the NFBC project center. The authors would also like to thank Mr. Jari Päkkilä for designing the software (electronic patient file) for this cohort study. NFBC 1966 received financial support from University of Oulu Grant no. 24000692,Oulu University Hospital Grant no. 24301140 and ERDF European Regional Development Fund Grant 
no. 539/2010 A31592. This study was supported by a grant from the Finnish Dental Association Apollonia and Colgate Gaba. The funders had no role in the development of the study design, data collection and analysis, the decision to publish, or the preparation of the manuscript. The authors alone are responsible for the content and writing of the paper. Study design: VuA, MLL, AL, LT. Performed the clinical examination: MLL,HK. Performed the 3D model analysis: RP, HS, ViA. Analyzed the data: ViA, RP, HS, PP, VuA, MLL. Wrote the paper: ViA, HS, RP, VuA, MLL, PP, HK, AL, LT.

\section{References}

[1] R.P. Shellis, M. Addy, The interactions between attrition, abrasion and erosion in tooth wear, Monogr. Oral Sci. 25 (2014) 32-45.

[2] P. Wetselaar, M.J. Wetselaar-Glas, M. Koutris, C.M. Visscher, F. Lobbezoo, Assessment of the amount of tooth wear on dental casts and intra-oral photographs, J. Oral Rehabil. 43 (2016) 615-620.

[3] D. Bartlett, C. Ganss, A. Lussi, Basic Erosive Wear Examination (BEWE): A new scoring system for scientific and clinical needs, Clin. Oral Invest. 12 (2008) 6568.

[4] V. Alaraudanjoki, M.L. Laitala, L. Tjaderhane, P. Pesonen, A. Lussi, V. Anttonen, Association of erosive tooth wear and dental caries in Northern Finland Birth Cohort 1966 - an epidemiological cross-sectional study, BMC Oral Health 17 (2016), doi:http://dx.doi.org/10.1186/s12903-016-0232-x.

[5] B. Dixon, M.O. Sharif, F. Ahmed, A.B. Smith, D. Seymour, P.A. Brunton, Evaluation of the basic erosive wear examination (BEWE) for use in general dental practice, Br. Dent J. 213 (2012), doi:http://dx.doi.org/10.1038/sj. bdj.2012.670.

[6] L.H. Hove, A. Mulic, A.B. Tveit, K.R. Stenhagen, A.B. Skaare, I. Espelid, Registration of dental erosive wear on study models and intra-oral photographs, Eur. Arch. Paediatr. Dent. 14 (2013) 29-34.

[7] A. Mulic, A.B. Tveit, N.J. Wang, L.H. Hove, I. Espelid, A.B. Skaare, Reliability of two clinical scoring systems for dental erosive wear, Caries Res. 44 (2010) $294-$ 299.

[8] M.K. Al-Omiri, M.G. Sghaireen, B.K. Alzarea, E. Lynch, Quantification of incisal tooth wear in upper anterior teeth: Conventional vs new method using toolmakers microscope and a three-dimensional measuring technique, J. Dent. 41 (2013) 1214-1221.
[9] P. Wetselaar, F. Lobbezoo, M. Koutris, C.M. Visscher, M. Naeije, Reliability of an occlusal and nonocclusal tooth wear grading system: clinical use versus dental cast assessment, Int. J. Prosthodont. 22 (2009) 388-390.

[10] K. Baroudi, S.N. Ibraheem, Assessment of chair-side computer-aided design and computer-aided manufacturing restorations: a review of the literature, J. Int. Oral Health 7 (2015) 96-104.

[11] R. DeLong, Intra-oral restorative materials wear: rethinking the current approaches: how to measure wear, Dent. Mater. 22 (2006) 702-711.

[12] Northern Finland Birth Cohort Studies. http://www.oulu.fi/nfbc/, 2016 (accessed 22.9.16).

[13] J.R. Landis, G.C. Koch, An application of hierarchical Kappa type statistics in the assessment of majority agreement among multiple observers, Biometrics 33 (1977) 363-374.

[14] A. Van'T Spijker, C.M. Kreulen, E.M. Bronkhorst, N.H.J. Creugers, Assessment of early attrition using an ordinary flatbed scanner, J. Dent. 40 (2012) 603-608.

[15] R. Perry, G. Kugel, K.H. Kunzelmann, H.P. Flessa, D. Estafan, Composite restoration wear analysis: conventional methods vs. three-dimensional laser digitizer, J. Am. Dent. Assoc. 131 (2000) 1472-1477.

[16] A.B. Meireles, A.W. Vieira, L. Corpas, B. Vandenberghe, F.S. Bastos, P. Lambrechts, et al., Dental wear estimation using a digital intra-oral optical scanner and an automated 3D computer vision method, Comput. Methods Biomech. Biomed. Eng. 19 (2016) 507-514.

[17] R.C. Olley, R. Wilson, D. Bartlett, R. Moazzez, Validation of the basic erosive wear examination, Caries Res. 48 (2014) 51-56.

[18] V. Margaritis, E. Mamai-Homata, H. Koletsi-Kounari, A. Polychronopoulou, Evaluation of three different scoring systems for dental erosion: a comparative study in adolescents, J. Dent. 39 (2011) 88-93.

[19] Y. Vered, A. Lussi, A. Zini, J. Gleitman, H.D. Sgan-Cohen, Dental erosive wear assessment among adolescents and adults utilizing the basic erosive wear examination (BEWE) scoring system, Clin. Oral Invest. 18 (2014) 1985-1990.

[20] R.T. Kono, G. Suwa, T. Tanijiri, A three-dimensional analysis of enamel distribution patterns in human permanent first molars, Arch. Oral Biol. 47 (2002) 867-875.

[21] T.M. Smith, A.J. Olejniczak, D.J. Reid, R.J. Ferrell, J.J. Hublin, Modern human molar enamel thickness and enamel-dentine junction shape, Arch. Oral Biol. 51 (2006) 974-995.

[22] W.P. Holbrook, C. Ganss, Is diagnosing exposed dentine a suitable tool for grading erosive loss? Clin. Oral Invest. 12 (2008) 33-39.

[23] T. Haketa, K. Baba, S. Akishige, K. Fueki, K. Kino, T. Ohyama, Accuracy and precision of a system for assessing severity of tooth wear, Int. J. Prosthodontics 17 (2004) 581-584. 